\title{
A Group's Level of Defensive Functioning Affects Individual Outcomes in Group Psychodynamic-Interpersonal Psychotherapy
}

Q1

Q2

\author{
Samantha Carlucci ${ }^{1,2}$, Livia Chyurlia ${ }^{1,2}$, Michelle Presniak ${ }^{3}$, Nancy Mcquaid ${ }^{2}$, James C. Wiley ${ }^{4}$, \\ Stephanie Wiebe ${ }^{2,5}$, Robert Hill ${ }^{2}$, Camille Garceau ${ }^{2}$, Danielle Baldwin ${ }^{2}$, Chloe Slowikowski ${ }^{5}$, \\ Iryna Ivanova $^{6}$, Renee Grenon ${ }^{2}$, Louise Balfour ${ }^{2}$, and Giorgio A. Tasca ${ }^{1,2}$ \\ ${ }^{1}$ Clinical Epidemiology Program, Ottawa Hospital Research Institute, Ottawa, Ontario, Canada \\ ${ }^{2}$ School of Psychology, University of Ottawa \\ ${ }^{3}$ Ottawa Neurofeedback Centre, Kanata, Ontario, Canada \\ ${ }^{4}$ Carleton University \\ ${ }^{5}$ Saint Paul University \\ ${ }^{6}$ Dr. Ivanova Psychology \& Associates, Ottawa, Ontario, Canada
}

\begin{abstract}
We used an actor-partner interdependence model (APIM) to study the association between the individual group member and other group therapy members' defensive functioning on an individual group member's treatment outcome. We hypothesized that (a) more adaptive individual defensive functioning at pretreatment will be significantly related to better treatment outcomes (i.e., lower binge-eating and interpersonal distress) at 6 months post-treatment; and (b) more adaptive other group members' defensive functioning at pretreatment will be significantly related to better treatment outcomes at 6 months post-treatment Participants $(N=136)$ were individuals with BED enrolled in group psychodynamic-interpersonal psychotherapy (GPIP). Participants completed attachment interviews and were assessed on interpersonal distress and days binged at pre-treatment and 6 months post-treatment. The interview audio recordings were transcribed and used to code defensive functioning. We found that individual overall defensive functioning (ODF) scores at pretreatment were not significantly associated with binge-eating frequency or interpersonal distress at 6 months post-treatment. Other group members' mean ODF scores at pretreatment were significantly associated with individual interpersonal distress at 6 months post-treatment. However, the other group members' mean ODF scores were not significantly associated with individual binge-eating outcomes at 6 months post-treatment. Defensive functioning of other members of a therapy group may be particularly important for improving interpersonal functioning in individuals with BED.
\end{abstract}

\section{Clinical Impact Statement}

Question: We studied the impact of the individual group member's and other group therapy members' defense mechanisms on an individual group member's treatment outcome (i.e., binge-eating frequency and interpersonal/social functioning). Findings: When other members of a therapy group used more adaptive defense mechanisms, the individual group member's interpersonal functioning improved; but

(c) 2021, American Psychological Association. This paper is not the copy of record and may not exactly replicate the final, authoritative version of the article. Please do not copy or cite without authors' permission. The final article will be available, upon publication, via its DOI: 10.1037/pst0000423

Nancy Mcquaid (DD https://orcid.org/0000-0003-0268-869X James C. Wiley (D) https://orcid.org/0000-0002-5049-573X Stephanie Wiebe (D) https://orcid.org/0000-0001-5865-4531 Robert Hill (iD https://orcid.org/0000-0002-1270-7789

Camille Garceau (iD) https://orcid.org/0000-0001-9514-5445

Giorgio A. Tasca (iD https://orcid.org/0000-0001-7596-0661

Samantha Carlucci played a lead role in data curation, formal analysis, writing of original draft and writing of review and editing and equal role in conceptualization, investigation and methodology. Livia Chyurlia played a supporting role in data curation and writing of review and editing and equal role in conceptualization and project administration. Michelle Presniak played a lead role in resources, supporting role in conceptualization and equal role in supervision and validation. Nancy Mcquaid played a supporting role in data curation and equal role in conceptualization and investigation. James C. Wiley played a supporting role in data curation, formal analysis and writing of review and editing. Stephanie Wiebe played a supporting role in writing of review and editing and equal role in data curation. Robert Hill played a supporting role in writing of review and editing and equal role in data curation. Camille Garceau played an equal role in data curation and writing of review and editing. Danielle Baldwin played a supporting role in writing of review and editing and equal role in data curation. Chloe Slowikowski played an equal role in data curation. Iryna Ivanova played a supporting role in data curation. Renee Grenon played a supporting role in data curation. Louise Balfour played a supporting role in conceptualization. Giorgio A. Tasca played a lead role in conceptualization, funding acquisition, investigation and supervision, supporting role in formal analysis and writing of review and editing and equal role in methodology.

Correspondence concerning this article should be addressed to Samantha Carlucci, School of Psychology, University of Ottawa, 136 Jean-Jacques Lussier, Vanier Hall, Ottawa, ON K1N 6N5, Canada. Email: scar1090@uottawa.ca 
when the group used less adaptive defense mechanisms, the individual's interpersonal functioning worsened. Meaning: The other group members' defense mechanisms impacted the individual member's interpersonal functioning. The group's characteristics are important for individual treatment outcomes. Next Steps: Future research should study the effect of defense mechanisms in other types of group therapy. Furthermore, therapists should consider the group's composition, as individual members who significantly differ from the group may not benefit from treatment.

Keywords: binge-eating disorder, defensive functioning, group therapy, interpersonal functioning, actor-partner interdependence model

Supplemental materials: https://doi.org/10.1037/pst0000423.supp

Defensive functioning is a psychological process used to manage internal conflict (Perry et al., 2009). It may play an important role in the treatment of binge-eating disorder (BED). However, there is little research on the impact of an individual group therapy member's and other members' level of defensive functioning on individual treatment outcomes. For example, Leslie, who tends to use more adaptive defense mechanisms, joined a therapy group in which the other group members on average had a lower level of defensive functioning. Jung, on the other hand, who tends to use less adaptive defense mechanisms, joined a group whose members on average engaged in a higher level of defensive functioning. Does the level of defensive functioning of the group of which Leslie or Jung are members have an impact on their individual outcomes?

\section{Defensive Functioning}

Defense mechanisms exist on a hierarchy ranging from high adaptive to low adaptive (i.e., maladaptive). The most widely recognized hierarchy of defensive functioning is the Defense Mechanism Ratings Scale (DMRS; Perry, 1990), which includes 30 individual defenses across seven levels (see Table 1 in Online Supplemental Material). It produces an overall defensive functioning (ODF) score from 1.00 to 7.00 to capture one's general level or adaptiveness of defensive functioning.

Maladaptive defensive functioning is common among those with greater psychopathology (Kipper et al., 2004). For example, Vitousek et al. (1991) found that individuals with eating disorders tend to employ more maladaptive defense mechanisms than healthy controls. Similarly, Carlucci et al. (2021b) found that women with BED used significantly fewer Obsessional defenses (i.e., higher adaptive defenses such as isolation, intellectualization, undoing) and significantly more Disavowal defenses (i.e., less adaptive defenses such as denial, rationalization) than women without BED. Despite this research by Carlucci et al. (2021b), the role of defensive functioning in the development and treatment of BED is understudied.

\section{Defensive Functioning and Binge-Eating Disorder}

Binge-eating disorder is characterized by repeated episodes of over-eating accompanied by a sense of a loss of control, but not accompanied by any compensatory behaviors (e.g., laxative misuse, self-induced vomiting; American Psychiatric Association [APA], 2013). Binge-eating disorder is comorbid with a variety of physical (e.g., diabetes, obesity; Bulik \& Reichborn-Kjennerud, 2003) and mental health conditions (e.g., substance abuse disorder, depression, anxiety disorders; Grilo et al., 2009), as well as interpersonal distress (Blomquist et al., 2012).

The interpersonal theory of binge-eating posits that binge-eating is a means of coping with negative affect that arises from interpersonal problems (Ivanova et al., 2015). Since those with BED tend to experience greater interpersonal distress (Blomquist et al., 2012), they may be less able to manage their emotions and resort to maladaptive coping strategies (e.g., binge-eating) to minimize their negative affect (Brugnera et al., 2018). However, instead of resolving feelings of anxiety or internal conflict, both maladaptive defense mechanisms and binge-eating may elicit additional feelings of guilt or shame. Hill et al. (2015) found that a linear improvement in defensive functioning during the course of group therapy mediated a decrease in binge-eating episodes post-treatment. These findings suggest that interpersonal-dynamic group therapy may reduce interpersonal distress, binge-eating, and maladaptive defensive functioning.

To address maladaptive interpersonal patterns and problematic defenses common in BED, Tasca et al. (2021) developed group psychodynamic-interpersonal psychotherapy (GPIP). It is delivered across 16 weekly 90-min sessions and is designed to reduce maladaptive interpersonal functioning and promote more adaptive attachments, affect regulation, and defensive functioning. Treatment trials indicate that GPIP is efficacious in treating BED (Tasca et al., 2006). Carlucci et al. (2021b) found improvements in defensive functioning from preto 6 months post-GPIP in a sample of women with BED compared to a no treatment control condition. However, no previous study disaggregated individual and group effects of defensive functioning when assessing its impact on treatment outcomes.

\section{Disaggregating Individual and Group Effects}

One can expect that each individual member will impact the group-as-a-whole, and that the group-as-a-whole will have a reciprocal impact on its individual members and their outcomes. Returning to our examples, Leslie and Jung may have very different experiences of group therapy, and their outcomes may differ not only because of their own levels of defensive functioning but also because of their respective groups' levels of defensive functioning. Yalom and Leszcz (2020) referred to this reciprocal influence as a spiral that represents either adaptive or maladaptive cycles of interaction and behaviors between individuals and groups. In an adaptive spiral, an individual positively impacts the other group members who, in turn, positively impact the individual's functioning. For example, in a study of males with human immunodeficiency virus (HIV), an individual member's sense of hope improved and 
became more consistent with the groups' level of hope as treatment progressed (Kivlighan et al., 2016). In a maladaptive spiral, an individual may negatively impact the other group members' and, in turn, their own behavior. For example, Tasca et al. (2016) published a case report wherein an individual that was a poor fit to their therapy group hindered her own treatment progress and that of other group members.

Hence, to gain a more nuanced understanding of group psychotherapy and the factors that contribute to successful treatment, researchers must disaggregate the individual and other group members' impact. To study the reciprocal impact, researchers have adopted the actor-partner interdependence model (APIM; Kenny et al., 2002), which disaggregates the individual's effect (actor) from the other group members' effects (partner) on the individual's outcomes. Using the APIM, Lo Coco et al. (2012) found that the other group members' mean alliance rating was more strongly associated with individual symptom reduction than the individual's alliance ratings. These findings suggest that the individual's and other group members' characteristics can differentially impact an individual's experience of group processes and individual treatment outcomes (Kivlighan \& Narvaez, 2021). The APIM has not yet been applied to the study of defensive functioning in group therapy for BED. Hence, the present study aimed to test how a group's defensive functioning impacted individual outcomes in terms of binge-eating frequency and interpersonal distress. We hypothesized that (a) more adaptive individual defensive functioning at pretreatment will be significantly related to better individual treatment outcomes (i.e., lower bingeeating frequency and interpersonal distress) at 6 months posttreatment; and (b) more adaptive other group members' defensive functioning at pretreatment will be significantly related to better individual treatment outcomes (i.e., lower binge-eating and interpersonal distress) at 6 months post-treatment.

\section{Method}

\section{Participants}

Participants were 136 adults diagnosed with BED recruited from two studies: (a) a randomized-controlled trial of participants with BED assigned to GPIP ( $n=35$; Tasca et al., 2019), and (b) an uncontrolled treatment study in which women with BED received GPIP ( $n=101$; Tasca et al., 2013). The participants' mean age was 44.65 years $(S D=12.00)$, and their mean BMI was $37.87 \mathrm{~kg} / \mathrm{m}^{2}$ $(S D=7.44)$. The participants were predominantly female $(96.3 \%)$, English-speaking (82.7\%), White (91.6\%), employed $(80.0 \%)$, college or university educated $(51.1 \%)$, and reported an annual family income of $\$ 60,000-\$ 69,999 \mathrm{CAD}$. A total of 17 therapy groups were formed across both studies. Each GPIP group consisted of five to 11 members led by one of 11 trained therapists. All therapists had prior experience conducting group therapy and were adequately adherent to the GPIP manual (see Tasca et al., 2019, 2013). Participants provided written informed consent prior to enrolment. The XX Research Ethics Board and XX approved of the original protocols and this study.

\section{Measures}

\section{Diagnosis and Binge-Eating Episodes}

We diagnosed BED using the Structured Clinical Interview (SCID; First et al., 2007) for the Diagnostic and Statistical Manual of Mental Health (DSM; APA, 2000). The SCID has good inter-rater reliability with an observed kappa coefficient of 0.77 (Zanarini et al., 2000). We assessed binge-eating via the Eating Disorder Examination (EDE; Fairburn \& Cooper, 1993), which is a semistructured interview that in part assesses binge-eating frequency in the last 28 days. The EDE had good inter-rater reliability with intra-class correlation coefficients ranging from 0.91 to 0.99 (Tasca et al., 2019, 2013).

\section{Interpersonal Distress}

The Inventory of Interpersonal Problems-64 (IIP-64: Horowitz et al., 1988) is a self-report questionnaire that evaluates interpersonal dysfunction and distress. Participants rated each item on a 5point scale $(0=$ not at all $; 4=$ extremely $)$, with higher total scores indicating greater interpersonal distress. The IIP-64 total score demonstrated good reliability within our samples, with a mean inter-item correlation of 0.42 (Tasca et al., 2013) and a coefficient $\alpha$ of 0.96 (Tasca et al., 2019).

\section{Rating Defensive Functioning From Adult Attachment Interviews}

The Adult Attachment Interview (AAI: Main et al., 2002) is a semistructured interview whereby participants are asked to recall childhood memories of primary caregiver(s). The present study did not make use of the AAI to rate attachment mental states, rather the interviews provided the transcripts and audio recordings for DMRS coding. Despite the retrospective nature of the AAI, the coders used the DMRS to rate current instances of defensive functioning. This method of coding DMRS demonstrated good inter-rater reliability, with intra-class correlations ranging from .64 to .89 within our samples (Carlucci et al., 2021a).

\section{Procedure}

Participants were recruited through a tertiary care eating disorders clinic or self-referred in response to advertisements. A research coordinator screened participants for eligibility criteria and for the presence of BED using the EDE (Fairburn \& Cooper, 1993). All participants received a pre-group preparation session and 16 weekly sessions of GPIP. They also completed the following measures at two time-points (i.e., pre- and 6 months post-treatment): the AAI which was coded with the DMRS, the IIP-64, and the EDE to assess days binged in the past 28 days. Hence, the present study included (a) a re-analysis of previously published outcome data on interpersonal functioning and binge-eating frequency (Tasca et al., 2013; Tasca et al., 2019), and (b) new data on defensive functioning coded by the DMRS from the AAI audio recordings. We only included pretreatment defensive functioning in this study, as it was the most representative of the potential impact of defensive functioning as it operated early in the therapy groups.

\section{Statistical Analyses}

We ran a two-level hierarchical linear model (HLM) to assess the impact of individual defensive functioning and other group members' mean level of defensive functioning on two treatment outcomes, binge-eating frequency and interpersonal distress. Given the 
structure of our data (i.e., individuals nested within therapy groups, nested within therapists), we first considered a three-level model. However, there was minimal variability between therapists $(0.004 \%$ to $0.02 \%$ ), as assessed from the variance components of completely unconditional three-level models; therefore, we proceeded with a two-level model. We used an APIM (Kenny et al., 2002) to study the impact of the individual and the therapy group's mean level of defensive functioning on the individual's outcomes. We subtracted the individual's score from the group when calculating the group mean relative to that individual, so that the group's mean score (partner) was independent of any individual's score (actor). We ran the HLM with full-maximum likelihood estimation and included a random intercept in each model. Level 1 modeled variability between individuals within groups, and level 2 modeled parameter variability between groups. For significant effects of parameters, we assessed the amount of within group variance accounted for by adding a level 1 predictor to the model by calculating a pseudo $R^{2}$ statistic. The full model testing the APIM is depicted in the Online Supplemental Material.

\section{Results}

\section{Data Screening}

Four participants had missing pre-treatment ODF scores. To impute these values, we used the 1-nearest neighbour (1-NN) method (Beretta \& Santaniello, 2016). Analyses with and without these missing cases were almost identical, so we kept these data. Two participants had missing data for IIP total at pretreatment and were deleted from the analyses of the IIP. There was no missing data for binge-eating at pre-treatment.

The percentage of missing data at 6 months post-treatment for bingeeating was $8.09 \%$, and for the IIP-64 was $38.97 \%$. We found that the missingness pattern was not associated with pre-treatment values of binge-eating ( $p=.799)$ or IIP-64 $(p=.512)$, and so we assumed that the data were missing at random. We did not impute missing values for binge-eating or interpersonal functioning at 6 months post-treatment. Binge-eating scores at 6 months post-treatment were significantly positively skewed. To normalize the distribution, we added a constant to binge-eating at pre-treatment and 6 months post-treatment and applied a log transformation. There were no univariate outliers.

\section{Main Analyses}

Our first hypothesis was not supported. We did not find an effect of individual ODF scores when controlling for adjusted mean group values (actor effects) for binge-eating frequency, $\beta_{20}=0.25, S E=$ $0.28, t(105)=0.90, p=.372$, pseudo $R^{2}=0.002$, or interpersonal distress, $\beta_{20}=1.54, S E=1.45, t(63)=1.06, p=.294$, pseudo $R^{2}=0.03$.

Our second hypothesis was partially supported. We found that adjusted mean group ODF scores (partner effects) were significantly associated with individual interpersonal distress 6 months posttreatment after controlling for pre-treatment IIP scores and the individual's ODF score, $\beta_{30}=-31.99, S E=11.93, t(63)=$ $-2.68, p=.009$, pseudo $R^{2}=0.03$. That is, when other members of a group on average had more adaptive defensive functioning at pre-treatment, the individual group member reported less (i.e., more improvement in) interpersonal distress at 6 months post-treatment.
Conversely, lower mean group defensive functioning at pretreatment was related to poorer individual outcomes on interpersonal distress at 6 months post-treatment. Adding the adjusted group mean scores at level 1 of the model accounted for $4.6 \%$ of the withingroup variance in interpersonal distress outcomes. Other group members' mean ODF scores at pretreatment were not significantly associated with individual binge-eating frequency at 6 months posttreatment, $\beta_{30}=-0.14, S E=0.19, t(105)=-0.72, p=.473$, pseudo $R^{2}=0.002$.

\section{Discussion}

The goal of this study was to assess whether an individual's level of defensive functioning and the group context of defensive functioning early in group therapy were each uniquely related to individual treatment outcomes (i.e., binge-eating frequency and interpersonal distress) at 6 months post-treatment for those with BED receiving GPIP. Our first hypothesis was not supported. Individual defensive functioning at pre-treatment (actor effect) did not significantly impact individual binge-eating or interpersonal distress at 6 months post-treatment. These findings diverge from those of Hill et al. (2015), who found that change in defensive functioning rated across multiple sessions of group therapy was significantly associated with change in binge-eating frequency immediately post-treatment. It is possible that change in defensive functioning during the life of a group may play a more important role than pre-treatment levels in determining outcomes at post-treatment. The present study also focused on longer term outcomes occurring some 10-12 months after the pretreatment assessment of defensive functioning, and so the effects of those initial ODF levels may have dissipated.

Our second hypothesis was partially supported. Other group members' average level of defensive functioning at pretreatment (partner effect) was significantly associated with improvements in an individual's interpersonal distress at 6 months post-treatment. That is, when other group members on average had more adaptive defensive functioning at pretreatment individual group members reported greater improvements in interpersonal distress at a followup assessment, and when other group members had less adaptive defensive functioning individual group members reported poorer interpersonal distress 6 months post-treatment. The effect of other group members' baseline defensive functioning was independent of an individual member's own baseline defensive functioning. These findings are consistent with Lo Coco et al. (2012) who found that other group members' perception of the therapeutic alliance to their group was a better predictor of treatment outcome than a member's rating of their own alliance to the group. Kivlighan (2011) also found that other group members' perception of therapeutic factors (e.g., altruism, cohesion, interpersonal learning) was a better predictor of evaluation of a session than the individual's own experience.

These findings emphasize the importance of disaggregating individual and other group member characteristics in clinical practice and research. They also suggest that the group context is particularly important for some individual treatment outcomes. Patients may experience vicarious learning, model themselves after their peers, and receive interpersonal feedback (Yalom \& Leszcz, 2020). As such, clinicians should promote a functional and supportive group environment rather than focusing primarily on the functioning of the individual in group psychotherapy. To illustrate using the case examples from the start of this study, a group therapist 
might be most helpful to Leslie if the therapist worked to improve the group's average level of defensive functioning. And a group therapist might be most helpful to Jung if the therapist leveraged the group's higher level of defensive functioning.

We found that group defensive functioning did not predict individual outcomes of binge-eating at 6 months post-treatment. One possible explanation for the inconsistent ODF partner effects on binge-eating and interpersonal distress may be a function of the data collection procedure. In this study, coders rated defensive functioning from AAIs, which elicited defenses through questions on interpersonal relationships (e.g., child-parent relationships). Therefore, we might expect a stronger link between defensive functioning coded from AAIs and interpersonal functioning than binge-eating. This has implications for clinical practice such as patient assessment for the purpose of group composition. Tasca et al. (2021) emphasized that the individual's level of psychological functioning, and not necessarily DSM-5 diagnosis (APA, 2013), should be the basis for determining group composition. Groups with lower aggregate levels of defensive functioning may require therapists to put a greater emphasis on the interpersonal and intrapersonal stressors within and outside of the group that are caused by lower-level defenses like splitting, denial, and projective identification. Furthermore, Tasca et al. (2021) suggested that patients who are too divergent from the rest of the group on level of defensive functioning may not benefit from treatment in that group.

\section{Limitations \& Future Directions}

This study had several limitations. First, our study was underpowered (Huta, 2014) and findings should be interpreted with caution. We recommend that future studies consider using a Monte Carlo simulation test (Muthén \& Muthén, 2002) to determine sample size and statistical power prior to data collection. Second, there was a lack of diversity or representation in our study sample: most of our participants were White and female, despite recent literature suggesting that the prevalence of BED is comparable across Latin Americans, African Americans, and White Americans (Marques et al., 2011), and that males account for $40 \%$ of Americans diagnosed with BED (National Eating Disorders Association, 2018). Thus, our findings should be replicated with a more diverse sample. Third, we only included participants who completed treatment and completed measures at both pretreatment and 6 months post-treatment. The data were missing at random, and so the parameters were likely reliable. However, the amount of missing data, especially for the IIP64, might raise further concerns about reduced statistical power (McNeish, 2017). Hence, generalizing the results to participants who did not complete treatment and/or did not provide data at 6 months follow-up should be done with caution. Fourth, to generalize or compare these findings to other interventions, future research should examine the effect of the level of defensive functioning in other types of group psychotherapy. Fifth, although the AAI is a valid measure of current attachment states of mind regarding early attachment experiences (George et al., 1985), it may not have been sufficient to elicit current defensive functioning.

\section{Conclusion}

To the best of our knowledge, this is the first study to apply an APIM to assess the impact of individual and other group members' defensive functioning on treatment outcomes (i.e., binge-eating frequency and interpersonal distress) of individuals with BED in the context of GPIP. This study suggests that the social and psychological context of the therapy group as indicated by the group's level of defensive functioning is particularly important for improving interpersonal problems in individuals with BED. Referring to our fictional group therapy example at the outset, it is likely that Leslie and Jung's interpersonal outcomes will be determined in part by the average level of defensive functioning among the other members of their therapy group. And the group will exert an effect that is independent of Leslie and Jung's own level of defensive functioning.

\section{References}

American Psychiatric Association. (2000). Diagnostic and statistical manual of mental disorders (4th ed., text revision).

American Psychiatric Association. (2013). Diagnostic and statistical manual of mental disorders (5th ed.).

Beretta, L., \& Santaniello, A. (2016). Nearest neighbor imputation algorithms: A critical evaluation. BMC Medical Informatics and Decision Making, 16(Suppl. 3), Article 74. https://doi.org/10.1186/s12911-0160318-z

Blomquist, K. K., Ansell, E. B., White, M. A., Masheb, R. M., \& Grilo, C. M. (2012). Interpersonal problems and developmental trajectories of binge eating disorder. Comprehensive Psychiatry, 53(8), 1088-1095. https:// doi.org/10.1016/j.comppsych.2012.05.003

Brugnera, A., Lo Coco, G., Salerno, L., Sutton, R., Gullo, S., Compare, A., \& Tasca, G. A. (2018). Patients with Binge Eating Disorder and Obesity have qualitatively different interpersonal characteristics: Results from an Interpersonal Circumplex study. Comprehensive Psychiatry, 85, 36-41. https:// doi.org/10.1016/j.comppsych.2018.06.008

Bulik, C. M., \& Reichborn-Kjennerud, T. (2003). Medical morbidity in binge eating disorder. International Journal of Eating Disorders, 34 (Suppl.), S39-S46. https://doi.org/10.1002/eat.10204

Carlucci, S., Chyurlia, L., Presniak, M., Mcquaid, N., Wiebe, S., Hill, R., Wiley, J., Garceau, C., Baldwin, D., Slowikowski, C., Ivanova, I., Grenon, R., Balfour, L., Maxwell, H., \& Tasca, G. A. (2021a). Assessing defense mechanisms in binge-eating disorder: Validity and reliability of the defense mechanism rating scale (DMRS) coded from Adult Attachment Interviews. [Manuscript submitted for publication].

Carlucci, S., Chyurlia, L., Presniak, M., Mcquaid, N., Wiebe, S., Hill, R., Wiley, J., Garceau, C., Baldwin, D., Slowikowski, C., Ivanova, I., Grenon, R., Balfour, L., \& Tasca, G. A. (2021b). Change in defensive functioning following group psychodynamic-interpersonal psychotherapy in women with binge-eating disorder. [Manuscript submitted for publication].

Fairburn, C. G., \& Cooper, Z. (1993). The eating disorder examination. In C. G. Fairburn \& G. T. Wilson (Eds.), Binge eating: Nature, assessment, and treatment (12th ed., pp. 317-360). Guilford Press.

First, M. B., Spitzer, R. L., Gibbon, M., \& Williams, J. B. W. (2007). Structured clinical interview for DSM-IV Axis I disorders-patient edition $(S C I D-I / P)$. New York State Psychiatric Institute.[Biometrics Res].

George, C., Kaplan, N., \& Main, M. (1985). Adult attachment interview. [Unpublished manuscript]. University of California.

Grilo, C. M., White, M. A., \& Masheb, R. M. (2009). DSM-IV psychiatric disorder comorbidity and its correlates in binge eating disorder. International Journal of Eating Disorders, 42(3), 228-234. https://doi.org/10 .1002/eat.20599

Hill, R., Tasca, G. A., Presniak, M., Francis, K., Palardy, M., Grenon, R., Mcquaid, N., Hayden, G., Gick, M., \& Bissada, H. (2015). Changes in defense mechanism functioning during group therapy for binge-eating disorder. Psychiatry, 78(1), 75-88. https://doi.org/10.1080/00332747 .2015 .1015897 
Horowitz, L. M., Rosenberg, S. E., Baer, B. A., Ureño, G., \& Villaseñor, V. S. (1988). Inventory of interpersonal problems: Psychometric properties and clinical applications. Journal of Consulting and Clinical Psychology, 56(6), 885-892. https://doi.org/10.1037/0022-006X.56.6.885

Huta, V. (2014). When to use hierarchical linear modeling. The Quantitative Methods for Psychology, 10(1), 13-28. https://doi.org/10.20982/tqmp.10 .1.p013

Ivanova, I. V., Tasca, G. A., Hammond, N., Balfour, L., Ritchie, K., Koszycki, D., \& Bissada, H. (2015). Negative affect mediates the relationship between interpersonal problems and binge-eating disorder symptoms and psychopathology in a clinical sample: A test of the interpersonal model. European Eating Disorders Review, 23(2), 133-138. https:// doi.org/10.1002/erv.2344

Kenny, D. A., Mannetti, L., Pierro, A., Livi, S., \& Kashy, D. A. (2002). The statistical analysis of data from small groups. Journal of Personality and Social Psychology, 83(1), 126-137. https://doi.org/10.1037/0022-3514.83 .1 .126

Kipper, L., Blaya, C., Teruchkin, B., Heldt, E., Isolan, L., Mezzomo, K., Bond, M., \& Manfro, G. G. (2004). Brazilian patients with panic disorder: The use of defense mechanisms and their association with severity. Journal of Nervous and Mental Disease, 192(1), 58-64. https://doi.org/ 10.1097/01.nmd.0000106001.84359.0f

Kivlighan, D. M. (2011). Individual and group perceptions of therapeutic factors and session evaluation: An actor-partner interdependence analysis. Group Dynamics, 15(2), 147-160. https://doi.org/10.1037/a0022397

Kivlighan, D. M., Jr., \& Narvaez, R. C. (2021). Mutual influence in group psychotherapy: A review and application to group psychology. In The psychology of groups: The intersection of social psychology and psychotherapy research (pp. 191-206). American Psychological Association. https://doi.org/10.1037/0000201-011

Kivlighan, D. M., Jr., Paquin, J. D., Hsu, Y. K. K., \& Wang, L. F. (2016). The mutual influence of therapy group members' hope and depressive symptoms. Small Group Research, 47(1), 58-76. https://doi.org/10.1177/ 1046496415605638

Lo Coco, G., Gullo, S., \& Kivlighan, D. M., Jr. (2012). Examining patients' and other group members' agreement about their alliance to the group as a whole and changes in patient symptoms using response surface analysis. Journal of Counseling Psychology, 59(2), 197-207. https://doi.org/10 $.1037 / \mathrm{a} 0027560$

Main, M., Goldwyn, R., \& Hesse, E. (2002). Adult attachment scoring and classification systems. Department of Psychology, University of California at Berkeley.

Marques, L., Alegria, M., Becker, A. E., Chen, C. N., Fang, A., Chosak, A., \& Diniz, J. B. (2011). Comparative prevalence, correlates of impairment, and service utilization for eating disorders across US ethnic groups: Implications for reducing ethnic disparities in health care access for eating disorders. International Journal of Eating Disorders, 44(5), 412-420. https://doi.org/10.1002/eat.20787

McNeish, D. (2017). Missing data methods for arbitrary missingness with small samples. Journal of Applied Statistics, 44(1), 24-39. https://doi.org/ 10.1080/02664763.2016.1158246

Muthén, L. K., \& Muthén, B. O. (2002). How to use a Monte Carlo study to decide on a sample size and determine power. Structural Equation Modeling, 9(4), 599-620. https://doi.org/10.1207/S15328007SE M0904_8
National Eating Disorders Association. (2018). Statistics and research on eating disorders. https://www.nationaleatingdisorders.org/statistics-resea rch-eating-disorders

Perry, J. C. (1990). The defense mchanism rating scales (5th ed.). Cambridge Hospital.

Perry, J. C., Beck, S. M., Constantinides, P., \& Foley, J. E. (2009). Studying change in defensive functioning in psychotherapy using the Defense Mechanism Rating Scales: Four hypotheses, four cases. In R. A. Levy \& J. S. Albon (Eds.), Handbook of evidence-based psychodynamic psychotherapy: Bridging the gap between science and practice (pp. 121-153). Humana Press. https://doi.org/10.1007/978-1-59745-444-5_6

Tasca, G. A., \& Balfour, L. (2014). Attachment and eating disorders: A review of current research. International Journal of Eating Disorders, 47(7), 710-717. https://doi.org/10.1002/eat.22302

Tasca, G. A., Mcquaid, N., \& Balfour, L. (2016). Complex contexts and relationships affect clinical decisions in group therapy. Psychotherapy: Theory, Research, \& Practice, 53(3), 314-319. https://doi.org/10.1037/ pst0000071

Tasca, G. A., Koszycki, D., Brugnera, A., Chyurlia, L., Hammond, N., Francis, K., Ritchie, K., Ivanova, I., Proulx, G., Wilson, B., Beaulac, J., Bissada, H., Beasley, E., Mcquaid, N., Grenon, R., Fortin-Langelier, B., Compare, A., \& Balfour, L. (2019). Testing a stepped care model for binge-eating disorder: A two-step randomized controlled trial. Psychological Medicine, 49(4), 598-606. https://doi.org/10.1017/ S0033291718001277

Tasca, G. A., Mikail, S. F., \& Hewitt, P. L. (2021). Group psychodynamic interpersonal psychotherapy. American Psychological Association. https://doi.org/10.1037/0000213-000

Tasca, G. A., Ritchie, K., Conrad, G., Balfour, L., Gayton, J., Lybanon, V., \& Bissada, H. (2006). Attachment scales predict outcome in a randomized controlled trial of two group therapies for binge eating disorder: An aptitude by treatment interaction. Psychotherapy Research, 16(1), 106121. https://doi.org/10.1080/10503300500090928

Tasca, G. A., Ritchie, K., Demidenko, N., Balfour, L., Krysanski, V., Weekes, K., Barber, A., Keating, L., \& Bissada, H. (2013). Matching women with binge eating disorder to group treatment based on attachment anxiety: Outcomes and moderating effects. Psychotherapy Research, 23(3), 301-314. https://doi.org/10.1080/10503307.2012.717309

Vitousek, K. B., Daly, J., \& Heiser, C. (1991). Reconstructing the internal world of the eating-disordered individual: Overcoming denial and distortion in self-report. International Journal of Eating Disorders, 10(6), 647666. https://doi.org/10.1002/1098-108X(199111)10:6<647::AID-EA T2260100604>3.0.CO;2-T

Yalom, I. D., \& Leszcz, M. (2020). The theory and practice of group psychotherapy (6th ed.). Basic Books.

Zanarini, M. C., Skodol, A. E., Bender, D., Dolan, R., Sanislow, C., Schaefer, E., Morey, L. C., Grilo, C. M., Shea, M. T., McGlashan, T. H., \& Gunderson, J. G. (2000). The collaborative longitudinal personality disorders study: Reliability of axis 1 and 2 diagnoses. Journal of Personality Disorders, 14(4), 291-299. https://doi.org/10.1521/pedi.2000 .14.4.291

Received July 28, 2021

Revision received November 19, 2021 Accepted November 30, 2021 